\title{
Circulating cell-free DNA levels in Portuguese patients with psoriasis vulgaris according to severity and therapy
}

\author{
S. Coimbra, ${ }^{1,2}$ C. Catarino, ${ }^{1,3}$ E. Costa, ${ }^{1,3}$ H. Oliveira, ${ }^{4}$ A. Figueiredo, ${ }^{4}$ P. Rocha-Pereira ${ }^{1,5}$ and A. Santos-Silva ${ }^{1,3}$ \\ ${ }^{1}$ Instituto de Biologia Molecular e Celular (IBMC), Universidade do Porto, Rua Campo Alegre 823, Porto 4150, Portugal \\ ${ }^{2}$ CESPU, Instituto de Investigação e Formação Avançada em Ciências e Tecnologias da Saúde, Gandra-PRD, Portugal \\ ${ }^{3}$ Laboratório de Bioquímica, Departamento de Ciências Biológicas, Faculdade de Farmácia (FFUP), Universidade do Porto, Porto, Portugal \\ ${ }^{4}$ Serviço de Dermatologia, Centro Hospitalar e Universitário de Coimbra, Universidade de Coimbra, Coimbra, Portugal \\ ${ }^{5}$ Centro de Investigação em Ciências da Saúde (CICS), Universidade da Beira Interior, Covilhã, Portugal
}

\section{Summary}

\section{Correspondence}

Susana Coimbra and Alice Santos-Silva.

E-mail:ssn.coimbra@gmail.com and

assilva@ff.up.pt

\section{Accepted for publication}

11 November 2013

\section{Funding sources}

This study was supported by Fundação para a Ciência e Tecnologia (FCT: POCI/SAU - OBS/ 58600/2004) and Fundo Europeu de Desenvolvimento Regional (FEDER). FCT and FEDER had no role in the design and conduct of the study; in the collection, analysis and interpretation of data; or in the preparation, review or approval of the manuscript.

\section{Conflicts of interest}

None declared.

DOI 10.1111/bjd.12738
Background Inflammation has a key role in the pathogenesis of psoriasis. Circulating cell-free DNA (CFD) is a marker of tissue cell damage closely associated with inflammation.

Objectives We aimed to understand the relation of CFD levels with psoriasis severity, defined by the Psoriasis Area and Severity Index (PASI), with inflammation and with psoriasis therapy.

Methods Forty-six patients with psoriasis vulgaris were evaluated before (T0) and after 12 weeks (T12) of treatment with narrowband ultraviolet light B (NB-UVB; $\mathrm{n}=17)$, psoralen plus UVA (PUVA; $\mathrm{n}=20$ ) or topical therapy $(\mathrm{n}=9)$. We evaluated interleukin (IL)-6 and circulating CFD levels.

Results Compared with controls, at T0, patients presented significantly higher levels of circulating CFD. CFD presented a significant positive correlation with IL-6 and a trend towards a positive correlation with PASI. Multiple linear regression analysis identified IL-6 as an independent variable associated with CFD circulating levels. As shown by the PASI score, a trend towards higher values of CFD was observed in the severe psoriasis forms; moderate and severe psoriasis presented also significantly higher CFD values, compared with control. Both NB-UVB and PUVA treatments significantly decreased the levels of CFD.

Conclusions Patients with psoriasis, at the active stage of the disease, presented an increased inflammation associated with raised circulating CFD levels, which seem to be linked to psoriasis severity. Both NB-UVB and PUVA, anti-inflammatory therapies, were effective in decreasing CFD values. We propose that the evaluation of circulating CFD may provide a new biomarker to monitor psoriasis, its severity and its treatment.

\section{What's already known about this topic?}

- Circulating cell-free DNA (CFD) levels, a marker associated with inflammation, has never been evaluated in psoriasis.

\section{What does this study add?}

- At the active stage of psoriasis, patients presented an increased inflammation associated with raised circulating CFD levels, which seem to be linked to psoriasis severity.

- NB-UVB and PUVA, anti-inflammatory therapies, were effective in decreasing CFD values.

- The evaluation of circulating CFD may provide a new biomarker to monitor psoriasis, its severity and its treatment. 
Marked chronic inflammation is a hallmark of psoriasis, associated with a rise in several inflammatory biomarkers. ${ }^{1-4}$ Increasing psoriasis severity has been associated with an enhancement in the inflammatory response. ${ }^{3}$

Circulating cell-free DNA (CFD) appears following cell damage and DNA release, and its levels are increased in pathological processes characterized by marked inflammation. ${ }^{5-7}$ In patients on haemodialysis, CFD levels were associated with inflammation ${ }^{8}$ and positively correlated with interleukin (IL)6. ${ }^{7,8}$ We found that IL-6 levels are increased in psoriasis and correlate with the Psoriasis Area and Severity Index (PASI). ${ }^{9}$

Considering the inflammatory and hyperproliferative psoriasis features and that, as far as we know, CFD levels have not been evaluated in psoriasis, we thought it important to evaluate them.

Our aim was to quantify circulating CFD levels in a Portuguese psoriatic population, in order to search for a relationship with inflammation and severity of the disease, and to study the relation of CFD with the efficacy of the therapy used.

The protocol used was approved by the Committee on Ethics of the University Hospital of Coimbra, Portugal.

Forty-six patients with psoriasis vulgaris were clinically and analytically studied in the active phase of the disease (T0) and 12 weeks (T12) after starting treatment. Seventeen patients were treated with narrowband ultraviolet light B (NB-UVB), 20 with psoralen plus UVA (PUVA) and nine with topical therapy. The control group included 20 healthy volunteers without psoriasis or other skin disease and with normal haematological and biochemical values. Protocol treatments and inclusion/exclusion criteria used were reported previously. ${ }^{9}$

Circulating CFD was detected directly in serum samples using a rapid direct fluorescent assay, according to Goldshtein et al. ${ }^{10}$ IL- 6 levels were evaluated by enzyme-linked immunosorbent assay (Human IL-6 ELISA, Bender MedSystems, Vienna, Austria). Psoriasis severity was evaluated by PASI. ${ }^{11}$

Clinical and analytical data for controls and patients with psoriasis are presented in Table 1 . At the active stage of psori- asis, we found that exacerbation of the lesions and of the inflammatory process were associated with increasing CFD levels, compared with controls. After successful treatment, demonstrated by the significant PASI reduction (Table 1), CFD levels decreased significantly. As previously reported, ${ }^{9}$ at T0 we found that IL-6 levels were enhanced, and decreased significantly after treatment. The CFD increase seems to be linked to the inflammatory response, as CFD values were positively correlated with the inflammatory marker IL-6 (Fig. 1a), known to be raised in active psoriasis. Moreover, in multiple linear regression analysis (using stepwise selection) $\mathrm{Lg}_{10}$ IL-6 remained significantly associated with $\operatorname{Lg}_{10}$ CFD $(\beta=0.225$; $\mathrm{P}=0.0001)$, identifying IL-6 as an independent variable associated with CFD levels. CFD was reported to be able to selectively induce IL-6 production by monocytes, ${ }^{12}$ which may justify its association with the inflammatory response. Whether CFD is a consequence of inflammation in psoriasis, or itself contributes to the inflammatory process is not known. Leaking of DNA fragments from the nuclei of leucocytes was reported as the possible source of CFD in patients on haemodialysis. ${ }^{12}$ In psoriasis, the origin of CFD has not been identified and might result from different inflammatory cells involved in psoriasis lesions.

At T0, NB-UVB [17.2 (12.6-25.0); median (interquartile range)] and PUVA [24.9 (13.3-33.5)] patients presented significantly higher PASI $(\mathrm{P} \leq 0.001$ and $\mathrm{P} \leq 0.001$, respectively) than topical patients $[8 \cdot 1(6 \cdot 7-10 \cdot 5)]$; at T12, the topical group $[3.6(2 \cdot 6-6 \cdot 8)]$ presented significantly higher PASI scores $(P=0.023)$ than the PUVA group $[1.6(0 \cdot 8-3 \cdot 9)]$, but not significantly different from the NB-UVB group $[3 \cdot 1$ $(1 \cdot 6-6 \cdot 6)]$. According to the PASI score, all treatments were successful as a decrease was found for NB-UVB, PUVA and topical therapy $(\mathrm{P} \leq 0.001, \mathrm{P} \leq 0.001$ and $\mathrm{P}=0.008$, respectively). Patients treated with NB-UVB and PUVA showed, before treatment, significantly higher CFD values, compared with controls; patients undergoing topical therapy presented only a trend towards higher CFD values $(P=0 \cdot 085)$. After treatment, the CFD levels decreased significantly for patients

Table 1 Clinical and analytical data for controls and patients with psoriasis vulgaris, before (T0) and at end of therapy (T12)

\begin{tabular}{|c|c|c|c|}
\hline & \multirow[b]{2}{*}{ Control $(n=20)$} & \multicolumn{2}{|c|}{ Psoriasis vulgaris patients $(\mathrm{n}=46)$} \\
\hline & & T0 & T12 \\
\hline Age (years) & $42 \cdot 4 \pm 16 \cdot 3$ & $44 \cdot 6 \pm 15 \cdot 7$ & - \\
\hline $\operatorname{Sex}(F / M)$ & $11 / 9$ & $24 / 22$ & - \\
\hline Smoking habits (s/ns) & $5 / 15$ & $8 / 38$ & - \\
\hline BMI (range) $\left(\mathrm{kg} \mathrm{m}^{-2}\right)$ & $25 \cdot 6(23 \cdot 5-29 \cdot 6)$ & $28 \cdot 2(25 \cdot 2-29 \cdot 7)$ & - \\
\hline ToDsD (years) & - & $18 \cdot 8 \pm 11 \cdot 7$ & - \\
\hline PASI & - & $16 \cdot 6(10 \cdot 5-26 \cdot 6)$ & $2 \cdot 8(1.4-4 \cdot 8)^{\mathrm{c}}$ \\
\hline Cell-free DNA (range) $\left(\mathrm{ng} \mathrm{mL}^{-1}\right)$ & $336(247-475)$ & $518(370-701)^{\mathrm{a}}$ & $449(319-522)^{d}$ \\
\hline Interleukin-6 (range) $\left(\mathrm{pg} \mathrm{mL}^{-1}\right)$ & $0.45(0.21-0.66)$ & $1 \cdot 10(0 \cdot 60-2 \cdot 22)^{\mathrm{b}}$ & $0.95(0.36-1.61)^{\mathrm{e}, \mathrm{f}}$ \\
\hline
\end{tabular}

To compare control with patients at T0 and T12, we used the Mann-Whitney U-test; to evaluate differences between T0 and T12, we used the Wilcoxon test; measurements are expressed as mean $\pm \mathrm{SD}$, or as median values (interquartile ranges). s, smoker; ns, nonsmoker; BMI, body mass index; ToDsD, time of disease since diagnosis; PASI, psoriasis area and severity index. ${ }^{\mathrm{a} P}$ (T0 vs. control) $\leq 0.001$; ${ }^{\mathrm{b}} \mathrm{P}$ (T0 vs. control $) \leq 0.001 ;{ }^{\mathrm{C} P}(\mathrm{~T} 0$ vs. $\mathrm{T} 12) \leq 0.001 ;{ }^{\mathrm{d} P}(\mathrm{~T} 0$ vs. $\mathrm{T} 12) \leq 0.001 ;{ }^{\mathrm{e}} \mathrm{P}(\mathrm{T} 0$ vs.T12 $)=0.002 ;{ }^{{ }^{\circ}} \mathrm{P}(\mathrm{T} 12$ vs. control $)=0.015$ 


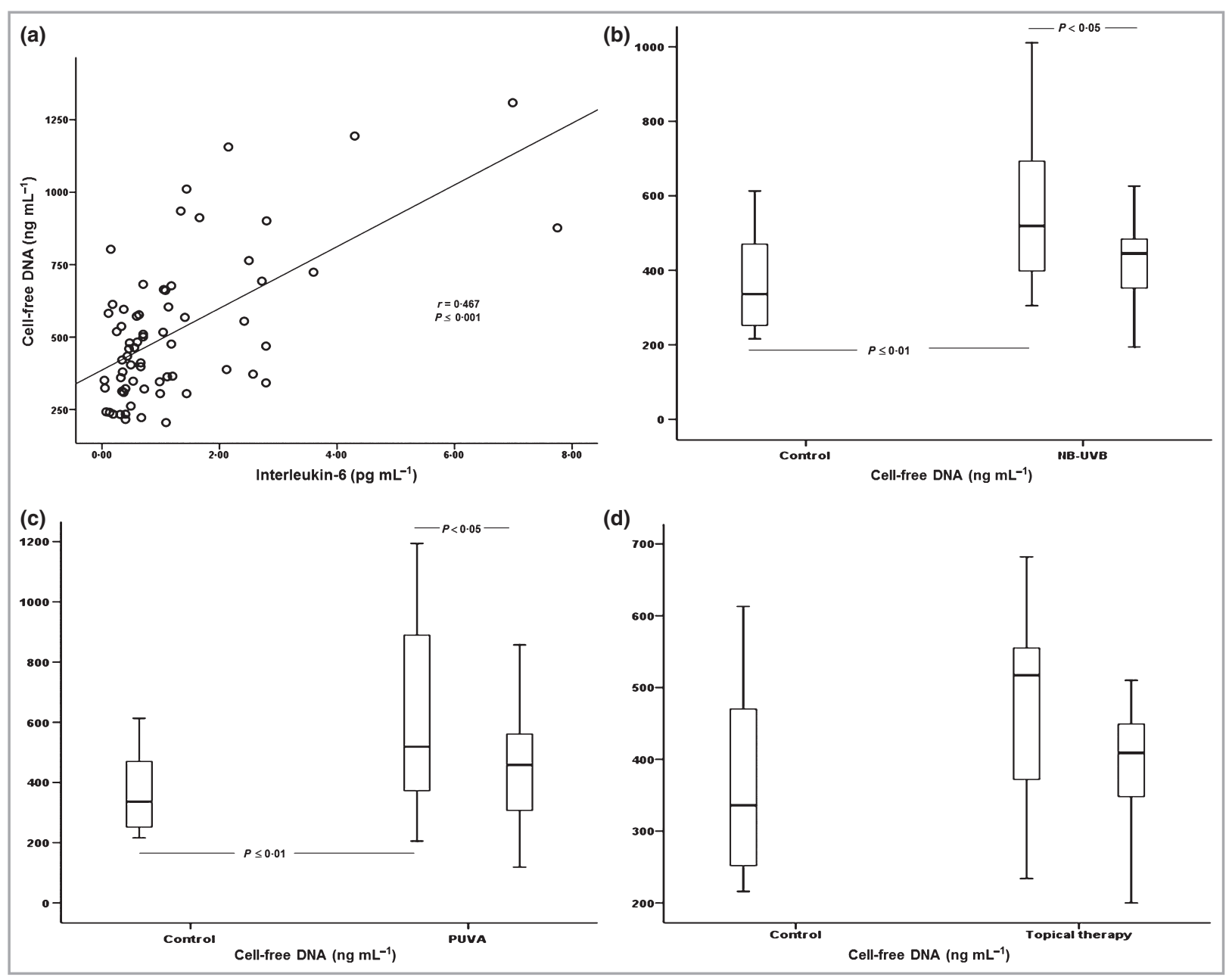

Fig 1. (a) Correlation found for psoriasis vulgaris patients between cell-free DNA (ng mL ${ }^{-1}$ ) and interleukin-6 (pg mL ${ }^{-1}$ ). Values of circulating cell-free DNA ( $\mathrm{n} \mathrm{mL}^{-1}$ ) for control and patients with psoriasis according to treatment with (b) narrowband ultraviolet light B (NB-UVB), (c) psoralen plus UVA (PUVA) and (d) topical therapy. The Spearman coefficient correlation was calculated. To compare groups according to therapy and severity, we used one-way ANova supplemented with Tukey post hoc test (after linearization of variables, when necessary).

treated with NB-UVB and PUVA and a trend towards lower values was found for topically treated patients (Fig. 1b-d). Concerning IL-6, a significant reduction was observed after treatment for PUVA group, as reported. ${ }^{9}$

The significant decrease in CFD levels observed after therapy strengthens its linkage to inflammatory cell damage; indeed, this reduction was associated with a decrease in inflammation and in psoriasis lesions, as shown by a decrease in IL-6 and PASI, respectively. PUVA and NB-UVB therapies, used to treat moderate and severe forms of psoriasis, are known to present anti-inflammatory and immunomodulatory activities, ${ }^{13}$ and to be associated with a reduction in inflammatory markers. ${ }^{3,4,9,14}$ They were clearly successful therapies, as confirmed by the significant decrease in PASI, and for both we observed similar modifications, a decrease in CFD levels, which is in accordance with a reduction in the inflammatory state. We found no significant changes in CFD with topical therapy. Topical agents, used for the mildest forms of psoriasis, act locally and seem to present a less marked anti-inflammatory activity.
According to the PASI score, at T0, 10 of the patients presented mild (score below 10), 18 moderate (score between 10 and 20) and 18 severe (score above 20) psoriasis. ${ }^{15}$ The severe and moderate forms of psoriasis presented significantly higher values of CFD, compared with controls $(P=0.012$ and $\mathrm{P}=0.001$, respectively), and a trend towards higher values was observed for the mild form. The same pattern was found for IL-6, as previously reported. ${ }^{9}$

Increasing psoriasis severity was associated with a significantly higher inflammatory degree, as shown by IL-6 values. Although circulating CFD presented only a trend towards higher values with increasing psoriasis severity $(r=0 \cdot 234$; $P=0 \cdot 117)$, we found a positive significant correlation with IL-6 ( $r=0.303 ; \quad P=0.041)$, suggesting an association of worsening of psoriasis and inflammation with an increase in inflammatory cell damage and, therefore, in circulating CFD. Indeed, compared with controls, patients in the NB-UVB and PUVA groups presented higher CFD values at T0 and, when compared with patients in the topical group, the NB-UVB and 
PUVA groups presented higher PASI scores. At T0, the topical group showed milder psoriasis, as defined by PASI, and did not present significant differences in CFD from the control group, before and after treatment. Further studies in a larger population are warranted, in order to confirm our data suggesting that circulating CFD levels are associated with the severity of psoriasis.

In summary, patients with psoriasis, at the active stage of the disease, present an increased inflammation associated with raised circulating CFD levels, which seem to be linked to psoriasis severity. Both NB-UVB and PUVA, anti-inflammatory therapies, were effective in decreasing CFD values. We propose that the evaluation of circulating CFD, using an assay that is easy to perform and not expensive, may provide a new biomarker to monitor psoriasis, its severity and its treatment.

\section{References}

1 Rocha-Pereira P, Santos-Silva A, Rebelo I et al. The inflammatory response in mild and in severe psoriasis. Br J Dermatol 2004; 150:917-28.

2 Chodorowska G, Wojnowska D, Juszkiewicz-Borowiec M. C-reactive protein and alpha2-macroglobulin plasma activity in mediumsevere and severe psoriasis. J Eur Acad Dermatol Venereol 2004; 18:180-3.

3 Coimbra S, Oliveira H, Reis F et al. C-reactive protein and leucocyte activation in psoriasis vulgaris according to severity and therapy. J Eur Acad Dermatol Venereol 2010; 24:789-96.

4 Coimbra S, Oliveira H, Reis F et al. Interleukin (IL)-22, IL-17, IL23, IL-8, vascular endothelial growth factor and tumour necrosis factor-alpha levels in patients with psoriasis before, during and after psoralen-ultraviolet A and narrowband ultraviolet B therapy. Br J Dermatol 2010; 163:1282-90.
5 Lo YM, Rainer TH, Chan LY et al. Plasma DNA as a prognostic marker in trauma patients. Clin Chem 2000; 46:319-23.

6 Lam NY, Rainer TH, Wong LK et al. Plasma DNA as a prognostic marker for stroke patients with negative neuroimaging within the first 24 h of symptom onset. Resuscitation 2006; 68:71-8.

7 Tovbin D, Novack V, Wiessman MP et al. Circulating cell-free DNA in hemodialysis patients predicts mortality. Nephrol Dial Transplant 2012; 27:3929-35.

8 Kohlova M, Ribeiro S, do Sameiro-Faria M et al. Circulating cellfree DNA levels in hemodialysis patients and its association with inflammation, iron metabolism, and rhEPO doses. Hemodial Int 2013; 17:664-7.

9 Coimbra S, Oliveira H, Reis F et al. Circulating adipokine levels in Portuguese patients with psoriasis vulgaris according to body mass index, severity and therapy. J Eur Acad Dermatol Venereol 2010; 24:1386-94.

10 Goldshtein H, Hausmann MJ, Douvdevani A. A rapid direct fluorescent assay for cell-free DNA quantification in biological fluids. Ann Clin Biochem 2009; 46(Pt 6):488-94.

11 Frederiksson T, Pettersson U. Severe psoriasis - oral therapy with a new retinoid. Dermatologica 1978; 157:238-44.

12 Atamaniuk J, Kopecky C, Skoupy S et al. Apoptotic cell-free DNA promotes inflammation in haemodialysis patients. Nephrol Dial Transplant 2012; 27:902-5.

13 Lebwohl M, Ali S. Treatment of psoriasis. Part 1. Topical therapy and phototherapy. J Am Acad Dermatol 2001; 45:487-98; quiz 499502 .

14 Coimbra S, Oliveira H, Neuparth MJ et al. Inflammatory markers of cardiovascular disease risk in Portuguese psoriatic patients: relation with narrow-band ultraviolet $\mathrm{B}$ and psoralen plus ultraviolet $\mathrm{A}$. Int J Dermatol 2014; 53:393-6.

15 Naldi L, Gambini D. The clinical spectrum of psoriasis. Clin Dermatol 2007; 25:510-18. 